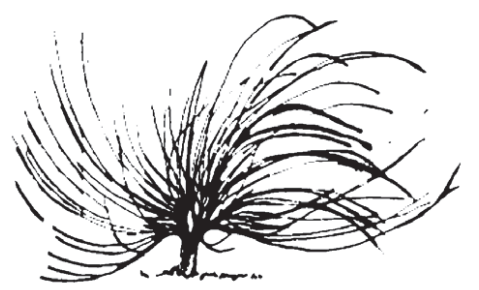

\title{
La Identidad Profesional Docente: Un Estudio en el Departamento de Lingüística de la Escuela de Filología, Lingüística y Literatura de la Universidad de Costa Rica
}

\author{
Cidalice Cerdas Benavides ${ }^{1}$ \\ Universidad Nacional \\ Heredia, Costa Rica \\ cidalice21@gmail.com
}

\begin{abstract}
Resumen
Este artículo tiene como objetivo analizar la identidad profesional docente del profesorado del Departamento de Lingüística de la Escuela de Filología, Lingüística y Literatura de la Universidad de Costa Rica. Para ello, se parte de la pregunta: ¿Cómo definen su identidad profesional los docentes que imparten lecciones en la carrera de Filología Española? En torno a este cuestionamiento se realiza un análisis de los aspectos fundamentales de la identidad profesional: las concepciones sobre la enseñanza y el aprendizaje; las estrategias empleadas, y los factores afectivos y motivacionales.
\end{abstract}

Palabras claves: Identidad profesional, Filología, enseñanza, concepciones sobre enseñanza, estrategias, factores afectivos.

Recibido: 16 de setiembre de 2013 - Aprobado: 22 de enero de 2014

1 Filóloga y docente. Egresada de la Maestría en Educación con énfasis en Docencia Universitaria de la Universidad Nacional 


\begin{abstract}
This article aims to analyze the professional identity of professors teaching in the Department of Linguistics at the School of Philology, Linguistics and Literature at the University of Costa Rica. In order to do so, it posits the question "How do professors providing lessons in Spanish Philology major define their identity?" Concerning the question, an analysis of the fundamental aspects of professional identity was executed taking into consideration: the conceptions of teaching and learning, strategies employed, as well as the related affective and motivational factors.
\end{abstract}

Key words: Professional identity, Philology, teaching, learning conceptions, strategies, affective factors

\title{
Introducción
}

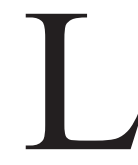

a docencia universitaria es una práctica a la que se llega por caminos que no siempre se relacionan con el estudio de la pedagogía, o para el caso de los adultos, la andragogía. A pesar de esto, los académicos construyen una identidad profesional que se vincula con una serie de concepciones, acciones y sentimientos con los cuales se identifican en el desempeño de su quehacer y que, por tanto, intervienen directamente en este.

Durante mucho tiempo se ha abogado por una mayor implementación de métodos innovadores en la educación superior. No obstante, siempre ha existido una gran dificultad para llevarlos a la práctica; esto no necesariamente como resultado de desconocimiento de las metodologías constructivistas, sino porque en gran parte dependiendo de las imágenes que un profesor tenga de sí mismo, de cómo se sienta con su trabajo y de las estrategias que conozca, empleará ciertos modelos pedagógicos, a pesar de que tenga conocimiento de otros.

El estudio de la identidad profesional docente ofrece la posibilidad de conocer las concepciones que los académicos tienen sobre cómo se enseña y aprende su profesión; de igual manera, explora las estrategias de enseñanza que ponen en acción, así como también, los factores afectivos y motivacionales que les afectan. Según Monereo (2010) estos se consideran los tres aspectos que conforman la identidad profesional. Diversos autores 
vinculan este tema a la formación de docentes, pues su comprensión llevaría a exponer aspectos asociados con la resistencia a las innovaciones educativas o la perpetuación de metodologías tradicionales (Monereo, 2010; Contreras, 2010; Contreras, Monereo y Badia, 2010).

En países como España o Chile se han desarrollado múltiples investigaciones sobre el tema, lo cual ha desembocado en la creación de nuevos modelos de formación docente inicial y continua. Esto con el fin de que los académicos tengan mayor conocimiento de los elementos que pueden influir en su práctica y sepan cómo ajustarse a los retos que encuentran en el aula universitaria.

En nuestro país, esta veta de estudio posibilita valiosas investigaciones que podrían llevar a mejoras tangibles no solo en las metodologías de enseñanza, lo cual beneficiaría tanto a educandos como a profesores; sino también en el control de factores emocionales que influyen en la labor de estos últimos.

El propósito fundamental de este trabajo fue analizar la identidad profesional docente y sus implicaciones en las prácticas educativas de los profesores del Departamento de Lingüística que imparten cursos de la carrera de Filología Española en la Escuela de Filología, Lingüística y Literatura de la Universidad de Costa Rica. Los objetivos específicos fueron: en primer lugar, determinar las concepciones que poseen los docentes del departamento en cuestión sobre cómo se enseña y aprende su materia; en segundo lugar, mencionar el tipo de estrategias que emplean en su práctica; por último, describir su percepción sobre los aspectos afectivos y motivacionales que afectan su desempeño.

La mayoría de los profesores del Departamento de Lingüística, con el cual se trabajó en este estudio, no tienen formación en docencia, sin embargo, se busca indagar en su identidad profesional docente y las implicaciones que esta tiene en el desarrollo de prácticas educativas para así conocer mejor los aspectos que influyen en su quehacer.

\section{Antecedentes}

El estudio de la identidad profesional docente ha cobrado fuerza en otros países, principalmente se destaca la labor del SINTE (Seminario Interuniversitario de Investigación en Estrategias de Enseñanza y Aprendizaje) en España. De este grupo se derivan diversos estudios 
sobre la identidad profesional docente, la enseñanza estratégica y las teorías implícitas de la enseñanza.

Monereo (2010) propone que para llevar a cabo cambios más significativos en la práctica docente es necesario que se modifique la identidad profesional, esto podría lograrse mediante el análisis de los incidentes críticos, entendidos estos como:

Sucesos acotados en el tiempo y en el espacio que, al superar un determinado umbral emocional del profesor, pone en crisis o desestabiliza su identidad-en-acción, de modo que para recuperar el control de la situación no basta con aplicar una estrategia local, sino que requiere cierta revisión de algunos aspectos de la identidad profesional. (p. 159)

Contreras, Monereo y Badia (2010) también profundizan en los incidentes críticos, específicamente aquellos ocurridos en las aulas de profesores universitarios. Estos autores recogen teoría sobre la importancia de estos incidentes para la formación académica, no obstante, apuntan que para que sean provechosos en estos procesos, se debe situar al docente como protagonista, para que así tenga un rol activo en la revisión y construcción de aprendizajes.

Los incidentes críticos, como hechos relevantes, ponen en crisis la identidad, por lo tanto, para superarlos se hace necesario utilizar mecanismos psicológicos profundos, lo cual abre una puerta para emplearlos como un recurso de formación docente. Otros modelos basados en procesos de reflexión, según estos autores, producirían mejoras pero no un cambio en las concepciones y sentimientos arraigados (Contreras, Monereo y Badia, 2010, p. 5).

Contreras (2010) realiza un estudio sobre identidad profesional en docentes universitarios de Chile. La autora se propone determinar cómo construyen su identidad cinco profesores que trabajan en programas de formación para académicos. Este estudio, al igual que el anterior, surge de la preocupación por los modelos de formación, en este caso permanente. Al respecto la autora afirma que la formación "debe ser diseñada e implementada en condiciones que aumenten las posibilidades de que los docentes desarrollen las competencias para construir conocimiento, afrontar incidentes y mejorar su enseñanza” (p. 2). 
En Costa Rica se ha llevado a cabo una serie de investigaciones sobre las concepciones de los docentes sobre la profesión y su función, la satisfacción de los acedémicos, y la didáctica en la educación superior; todos ellos se vinculan con la identidad profesional docente. A continuación se mencionan investigaciones que abordan el tema en estudio.

Piedra y Gutiérrez-Soto (2012) plantean una serie de cuestiones directamente relacionadas con aspectos de la identidad profesional docente, aunque el término no se menciona en su trabajo. En relación con las concepciones sobre cómo se enseña o aprende, afirman que los profesores tienen usualmente dos modelos: el primero creado a través de las opiniones, talleres o cursos de formación docente y los ideotipos promulgados por las universidades; el segundo es el que realmente implementan. La distancia entre cada modelo depende de distintos factores como: grado de motivación, tiempo asignado a las labores docentes y los recursos didácticos disponibles (p. 9).

Según estos mismos autores, el profesor universitario "no logra verse a sí mismo bajo la identidad de un docente, sino que se considera simplemente un profesional que imparte cursos sobre temas específicos, a menudo altamente especializados" (Piedra y Gutiérrez-Soto, 2012, p. 10). Los investigadores afirman que las motivaciones de los profesores e investigadores para ejercer la docencia incluyen "razones distintas a las de encontrar algún tipo de realización, satisfacción personal, deber social, placer o compromiso con la educación y la formación de los futuros profesionales" (Piedra y Gutiérrez-Soto, 2012, p. 7).

Piedra y Gutiérrez-Soto (2012) señalan la ausencia de grandes cambios en la enseñanza, pues una gran mayoría de docentes aún la concibe como la transmisión de información, principalmente mediante clases magistrales (p. 13). Asimismo, hay una tendencia a evitar el cambio cuando el profesor se siente cómodo o poco confrontado con el uso de un modelo pedagógico tradicional.

Estos autores dejan claro que los cambios en el ejercicio de la docencia pasan por la capacidad de reflexión que tengan los docentes sobre sus propias prácticas. Es a través de las concepciones del aprendizaje que los sujetos pueden cambiar en la medida en la que se toma conciencia de la importancia de sus propios procesos (Piedra y Gutiérrez-Soto, 2012, pp. 15-16).

Francis (2012) también considera algunos aspectos relacionados con el cambio y más específicamente con el papel de la didáctica como 
oportunidad para mejorar la enseñanza. Señala que los esfuerzos realizados por los docentes siguen distintos caminos: en algunos casos, reiteran las tradiciones que el docente ha observado durante su propia formación; mientras en otros, se convierten en propuestas divergentes que reconsideran el papel del docente y estudiante tradicional (p.76).

Según Francis (2012), la didáctica debe orientar la práctica de la docencia (pp. 80-82). La autora destaca el concepto de estrategia o guión el cual "hace referencia a un conjunto de acciones que se proyectan y se ponen en marcha de forma ordenada para alcanzar un determinado propósito" (p. 86). Por lo tanto, trazar objetivos es una fase sumamente importante en la planificación de la enseñanza.

Venegas (1995) busca responder cómo conceptualizan la función docente los profesores de las áreas de Ciencias Básicas y Ciencias Sociales. De su trabajo se concluye que hay una preponderancia de la docencia por sobre la investigación y la acción social y, a pesar de la necesidad de articular las tres, en el ámbito de acción están segmentadas (p. 136).

Aunque se reconoce la importancia de la docencia, se llama la atención sobre la preocupación por la formación pedagógica de los académicos, pues esto se vincula con el mejoramiento de los procesos educativos y la calidad de la educación (Venegas, 1995, p. 143). Paralelamente, resulta relevante que en este estudio también se apunte a que los profesores se sienten más profesionales que docentes universitarios (p. 122).

Venegas (1995) señala también que cada unidad académica posee elementos organizativos, intereses y problemas particulares, disciplinas y formas de trabajo que les otorgan identidad (p. 197). Además, dentro de esa diversidad cultural, cada una valora de manera distinta la formación pedagógica: mientras para unas unidades académicas es más importante, otras estiman más la formación profesional del cuerpo docente (p. 204).

En su trabajo Cubero (1996) aborda el tema de la satisfacción del personal docente, por lo tanto, toca aspectos afectivos y motivacionales. Su investigación, de corte cuantitativo, analiza los motivos que causan que personal docente del área de Ciencias Sociales se sienta satisfecho o insatisfecho. La autora concluye que entre los factores que influyen negativamente están: salario, cantidad de trabajo, la necesidad de capacitación, el difícil ascenso en el Régimen Académico. Como los factores de satisfacción se mencionan: las relaciones con los estudiantes, la seguridad del puesto. 
Álvarez, Arely, Gazel, Gutiérrez y Vargas (2010) elaboran una investigación cualitativa de tipo descriptivo sobre las percepciones de los docentes en educación inicial sobre su papel en la sociedad. Este trabajo destaca la importancia de la vocación en la satisfacción de los docentes, pues "para que haya una educación de calidad e integral, como se ha mencionado es fundamental que el personal docente se sienta bien consigo mismo y con el trabajo que realiza" (p. 4). Estos autores afirman que los factores que influyen en la auto percepción de los docentes son: la motivación, las condiciones laborales, la vocación y el prestigio social, entre otros (p. 4).

\section{La identidad profesional docente y sus aspectos}

La identidad profesional, según Monereo (2010), se define como:

Conjunto de representaciones relacionadas con la docencia que un profesor tiene de sí mismo, y que son bastante estables en el tiempo y bien delimitadas en cuanto a su contenido. Estas auto-representaciones afectarían a tres aspectos fundamentales: el rol profesional; la enseñanza y el aprendizaje y; los sentimientos asociados a la docencia. (p.157).

La revisión bibliográfica permitió conceptualizar mejor cada uno de los aspectos señalados por Monereo. En primer lugar, describiremos las concepciones sobre la enseñanza. En segundo lugar, las estrategias utilizadas por los docentes. Por último, definiremos los factores emocionales que afectan la labor del profesorado.

Macchiarola y Vogliotti (2003) apuntan que el estudio de las creencias, representaciones y concepciones sobre la enseñanza y el aprendizaje "permite la explicitación de los marcos de referencia por medio de los cuales los profesores perciben y procesan la información, analizan, dan sentido y orientan sus prácticas pedagógicas" (p.9).

Las concepciones sobre el aprendizaje y la enseñanza son analizadas desde diversos enfoques, los estudios de Pozo, Mateos, Pérez y Scheuer (2006) consideran que dichas concepciones se organizan como teorías implícitas. Este tipo de investigaciones parte de un contexto psicológico en el cual se valoran las creencias, representaciones y concepciones. Son una alternativa a los trabajos que privilegian los enfoques 
proceso-producto que buscan medir la relación entre la eficacia docente y los resultados del aprendizaje, y el desempeño de los alumnos (Macchiarola y Vogliotti, 2003, p. 2).

Las teorías implícitas ponen énfasis en los procesos mediadores entre la enseñanza y el aprendizaje. Pozo et al. (2006) proponen tres: teoría directa, teoría interpretativa y teoría constructiva. A continuación se describen brevemente.

La teoría directa tiene varias versiones. La primera es llamada extrema, pues centra su atención en los resultados o productos del aprendizaje, sin tomar en consideración los contextos particulares, ni el resultado de procesos relacionados con la actividad del aprendiz; sino que el aprendizaje ocurrirá por la exposición al contenido u objeto de estudio, pues desde una epistemología realista se concibe como una reproducción fiel de la información o modelo presentado (Pozo et al., 2006, p.120-121).

Una versión más elaborada vincula los resultados a las condiciones cuyo cumplimiento asegura el aprendizaje, este siempre será igual, independientemente de quién aprenda y cómo lo haga. Es prueba de la teoría directa "la creencia de muchos profesores que la reproducción de los contenidos enseñados es la mejor prueba de comprensión por parte de los alumnos" (Pozo et al., 2006, p. 121).

Desde la teoría interpretativa se parte de la existencia de una conexión entre los resultados del aprendizaje, los procesos y las condiciones en que se produce. Según esto la actividad del aprendiz permite el logro de un buen aprendizaje mediante la puesta en acción de ciertos procesos; además, para ello "es necesario reducir al mínimo las distorsiones indeseables" (Pozo et al., 2006, p. 122). La base epistemológica (igual que en la teoría anterior) es realista pues el aprendizaje se entiende como una reproducción de la realidad o de los modelos culturales. Sin embargo, también es clara "la necesidad de procesos intermedios entre las representaciones internas y la entrada de información" (Pozo et al., 2006, p. 122).

Dicha teoría también tiene distintas versiones. Una primera ve el actuar del aprendiz en aspectos manifiestos, por ejemplo, prácticas repetidas. Para Pozo et al (2006) esto implica la idea de que el aprendizaje "no es fácil ni inmediato, sino que consume tiempo y demanda esfuerzo deliberado" (p. 123). La teoría interpretativa puede aumentar su complejidad cuando se acumulan nuevos procesos mentales. Otras versiones asumen la acción del aprendiz en términos de procesos mentales: 
El núcleo explicativo reside en la intervención de procesos mentales que generan, conectan, amplían y corrigen representaciones internas (al "descubrir", recordar, relacionar, especificar, descartar), o que regulan las propias prácticas (a plantearse metas, evaluar los propios resultados y ajustar la ejecución). (Pozo et al., 2006, p. 123).

Dentro de este marco, el aprendizaje es un proceso, que puede articularse como bucles en los cuales el nuevo aprendizaje produce nuevos estados del conocimiento que producen a su vez nuevas condiciones para futuros aprendizajes.

Por otra parte, desde la teoría constructiva el aprendizaje implica una redescripción de los contenidos que trata e incluso de la propia persona que aprende. Por lo tanto, para apreciar los resultados es imprescindible considerar los cambios en los propios procesos representacionales del aprendiz incluyendo la manera de dar significado al objeto de aprendizaje como las metas de aprendizaje que se propone. (Pozo et al., 2006, p. 124).

La teoría constructiva considera la importancia que tienen los procesos metacognitivos en la regulación del aprendizaje que hace el aprendiz, quien es consciente de las condiciones en las que ocurre el conocimiento. Esta teoría va más allá de la conciencia de la participación de procesos o conocimientos previos en el aprendizaje, sino que además considera que estos se construyen. Se diferencia de las otras por sus principios epistemológicos, pues se caracteriza por asumir que:

Distintas personas pueden dar significado a una misma información de múltiples modos, que el conocimiento puede tener distintos grados de incertidumbre, que su adquisición implica necesariamente una transformación del contenido que se aprende y también del propio aprendiz, y que esta transformación puede producir incluso una innovación del conocimiento cultural. Desde el punto de vista ontológico y conceptual, la teoría constructiva se asienta sobre la noción del aprendizaje como sistema dinámico autorregulado que articula condiciones, procesos y resultados. (Pozo et al., 2006, p. 126). 
El segundo componente de la identidad profesional son las estrategias utilizadas por los docentes. Monereo et al. (1999) definen estrategia como una guía de las acciones por seguir que se hace de manera consciente e intencional, dirigida por un objetivo relacionado con el aprendizaje. Debe existir también una construcción personal "relacionada con la reflexión activa y consciente respecto a cuándo y por qué es adecuado un procedimiento o una técnica determinada, o respecto a las exigencias que tanto el contenido como la situación de enseñanza plantean a la hora de resolver la tarea" (p.12).

Un docente que emplea estrategias en su práctica busca provocar en sus estudiantes "aprendizaje estratégico", el cual garantiza que no solo se aprenda a utilizar unos determinados procedimientos sino que se pueda identificar cuándo y por qué recurrir a ellos y "en qué medida favorecen el proceso de resolución de una tarea" (Monereo et al., 1999, p. 11). Igualmente, los profesores también deben poseer la capacidad de identificar el momento, la razón y la forma de activar una estrategia ante determinada situación. Para tomar este tipo de decisiones, en primer lugar se hace necesario conocer cómo emplear los conocimientos procedimentales; en segundo lugar, es posible elegir cómo y cuándo ponerlos en acción, o bien optar entre unos y otros (Monereo et al., 1999, p.12).

Dentro del paradigma de la enseñanza estratégica, el profesor tendrá como funciones destacar sus objetivos, decidir las actividades por realizarse, clarificar la evaluación y proporcionar a los estudiantes mecanismos para favorecer también el aprendizaje de estrategias (Monereo et al., 1999, pág. 12). Es decir, más que emplear ciertas técnicas o procedimientos, deberá conducir a sus estudiantes a la adquisición de estrategias propias que pondrán en práctica cuando ejerzan su profesión, en este caso particular la Filología Española.

El tercer componente de la identidad profesional docente son los factores afectivos. Marchesi y Díaz (s. f.) afirman que:

Confianza y autoestima están íntimamente relacionadas y ambas constituyen el núcleo básico de la identidad profesional (...). Ambos sentimientos suponen haber interiorizado determinados objetivos, saber defenderlos y llevarlos a la práctica, manejarse con tranquilidad en las tareas educativas con los alumnos, compañeros y padres, sentirse capaz de enfrentarse a nuevos retos y situaciones problemáticas así como reconocer los propios 
errores, y aceptar sin angustia las dificultades que puedan vivirse en los procesos de cambio. (p. 10).

Estos son factores que influyen en el docente impulsándolo y provocando sus actuaciones tanto positivas como negativas (Monereo, 2010, p. 158), por lo tanto, no son elementos desconectados de la realidad vivida en el aula de clase, sino que tienen repercusiones tangibles que, como parte de una identidad profesional, afectan las prácticas educativas.

Luego de realizar esta descripción de los aspectos de la identidad profesional se procedió a hacer el diseño de la investigación para descubrir cómo construyen su identidad los docentes del Departamento de Lingüística que imparten cursos en la carrera de Filología Española.

\section{Metodología empleada}

Esta investigación se diseñó como un estudio cualitativo de tipo exploratorio. Como ya se ha dicho fue realizado en el Departamento de Lingüística de la Escuela de Filología, Lingüística y Literatura de la Universidad de Costa Rica. Fueron elegidos 6 participantes, todos los cuales imparten cursos de la carrera de Filología Española. De ellos, solamente uno tiene formación en pedagogía.

Para ahondar en la identidad profesional docente se emplearon dos técnicas: las entrevistas semiestructuradas, en las cuales se mantuvo una conversación con los participantes para obtener información sobre los aspectos de la identidad docente; $y$ un autoinforme que permitió recabar datos sobre aquello que hacen antes, durante y después de dar una clase.

La unidad de análisis seleccionada fue la identidad profesional docente, dentro de la cual incluimos las secuencias discursivas que hacen referencia a esta. Las categorías de análisis fueron tres: las concepciones sobre la enseñanza y aprendizaje, las estrategias usadas por los docentes y los factores afectivos y motivacionales que los afectan. Estas categorías surgieron mediante la revisión bibliográfica que fue elaborada previamente.

Luego de realizada la transcripción de los datos se procedió, tal como sugiere Cisterna (2005), a llevar a cabo un proceso de selección de la información obtenida, a través del cual se desechó aquello que no tenía relevancia o pertinencia desde los objetivos de la investigación (p. 9). Posteriormente se asignó a los segmentos significativos la 
unidad o las categorías apriorísticas mediante el programa Atlas ti en su versión 6.2. A partir de la información recopilada se elaboró una serie de cuadros que permitieron la comparación entre las respuestas de los distintos participantes y así sistematizarlas.

\section{Resultados}

El análisis de los datos se elaboró por cada una de las categorías. A continuación se presentan los resultados obtenidos mediante los dos instrumentos para cada una de dichas categorías. Se realizó también una confrontación con la teoría y la posición de la investigadora.

La primera categoría de análisis se refiere a las concepciones que poseen los docentes sobre su profesión, las demandas de su práctica y lo que significa enseñar y aprender su materia (Monereo, 2010, p.157).

Este grupo docente, en cuanto a las concepciones sobre su profesión, reveló dos puntos importantes: en primer lugar existe una fuerte tendencia a concebir su práctica en relación con las particularidades de su campo disciplinar y lo que ellos consideran que debe saber un filólogo sobre este; en segundo lugar, una fuerte consideración de las implicaciones de su trabajo en su vida personal, en forma de oportunidades y fuente de satisfacción.

Todos los consultados manifestaron que la docencia en la carrera de Filología Española tiene repercusiones muy fuertes pues la labor de los profesores desemboca para ellos en una adecuada preparación de los futuros profesionales. Asimismo, redundará en un posicionamiento crítico de los estudiantes. Por ejemplo, uno de los profesores manifiesta:

Considero que el español es un instrumento que tenemos todos los hablantes nativos que nos permite desarrollarnos en todas las áreas de la vida, y el filólogo, aparte de que está llamado a investigarlo, a conocerlo, a escudriñarlo, también está llamado a cuidarlo. Entonces para mí es muy importante la formación que demos a estos nuevos filólogos, tanto clásicos como españoles, en aras del mejor mantenimiento del español y de su desarrollo en general. (Entrevista 6)

Uno de los entrevistados afirma que la docencia 
es un buen lugar para desarrollar ciertos proyectos que uno tiene, también es un lugar en el que uno tiene conciencia política, digo política en el sentido aristotélico de la cosa... uno puede también legitimar ciertas ideas y ciertos proyectos que uno tiene con el público. (Entrevista 1)

Esto ejemplifica que las repercusiones de la profesión en la vida del académico son puntos importantes para valorar su trabajo y tener ciertas representaciones sobre él. Mediante su labor se puede desarrollar también la investigación y la acción social, áreas difíciles de ejercer fuera de la Universidad y que, además, les brindan una gran satisfacción.

Se percibe fuertemente el impacto que tiene para ellos su campo disciplinar, por cuanto conlleva una gran responsabilidad en la formación de los futuros profesionales pues desde la óptica de estos docentes es necesario

hacerle entender a los estudiantes que la lengua no es esa cosa llena de normatividad que les enseñan en el colegio o en la escuela con una metodología anticuada, inadecuada, que hace que la gramática sea aburrida, sino que es... la lengua es como un organismo vivo, en evolución, en constante evolución. (Entrevista 5).

Se evidencia, pues, una gran preocupación por sensibilizar a los educandos ante el objeto de estudio.

Vinculado con lo anterior, las representaciones de los participantes sobre las demandas y retos de la docencia en su área revelan que es primordial desarrollar un aprecio por el lenguaje, así como una actitud crítica frente a la gramática. Las principales funciones y responsabilidades destacadas son la preparación del docente para llevar "los conocimientos actualizados a los futuros formadores del área del lenguaje" (Entrevista 3); la formación de personas reflexivas, y la investigación en el campo.

Esta última responsabilidad, la investigación, se resalta fuertemente, debido a que se considera no de manera absoluta sino en su relación con la docencia pues son vistas como áreas complementarias e interdependientes:

No concibo un profesor universitario que simplemente se dedique a reproducir ideas por más sistemáticas que las haga, puede ser que sistematice muy bien sus lecturas, que sea muy buen 
docente, que tenga una prácticas didácticas buenísimas; pero si no es investigador, yo ahí tengo mis reparos para considerarlo un buen profesor universitario porque para mí si es claro que el docente universitario tiene que producir conocimiento no solo reproducirlo. (Entrevista 2).

También se muestra una preocupación por el desarrollo en los estudiantes de una visión crítica y reflexiva sobre el lenguaje pero a través de la teoría. En este caso el papel del docente se considera fundamental, pues debe propiciar ese proceso mediante el abordaje de los enfoques teóricos en el campo de estudio:

Pero, y ahí está la diferencia, en lo que debe hacer: debe basarse ese pensamiento crítico y esa reflexión sobre todo en una base mínima teórica (...) Entonces, enseñar, tal vez muchos hemos cometido el error de quedarnos en el nivel nada más de la transmisión de esa base teórica, pero ese es el nivel más básico. Sin que la persona sea capaz de dar el salto a partir de esa base teórica, hacer más, tomar posiciones propias, reformular, relacionar conectar, disociarse de x postura, sin que eso pase, no hubo educación, casi que información nada más. (Entrevista 2).

Las concepciones sobre la enseñanza y el aprendizaje (teorías implícitas) denotan principalmente una preocupación por la motivación que debe dar el docente para que el estudiante lleve a cabo su aprendizaje. Es decir, su papel es primordial para que el aprendiz desarrolle un conocimiento óptimo. Pues, para los participantes, el aprendizaje ocurre a partir del esfuerzo de los estudiantes, pero con la participación activa del docente:

Si usted ha venido a clases, si usted es buen estudiante, si usted es aplicado, si usted ha escuchado mis explicaciones, si usted ha aclarado sus dudas conmigo, a usted le va a ir bien, yo siempre veo esto y es un patrón correlacional sistemático: la gente que se esfuerza, o la gente que es inteligente de por sí sola, la gente que es brillante, la gente que interviene en clase, que me hace preguntas les va a ir bien en el examen. (Entrevista 5). 
Además, uno de los entrevistados destacó las características que a su entender posee el buen docente: "una persona preparada, una persona que tal vez haya estudiado, tenga un doctorado, ya sepa, ya haya tenido pesquisas de varios tipos en ambientes universitarios" (Entrevista 5). La labor del docente será de "motivar, despertar esa inquietud en las personas; pero, y ahí está la diferencia, en lo que debe hacer: debe basarse en ese pensamiento crítico y esa reflexión; sobre todo en una base mínima teórica..." (Entrevista 2).

El docente, según afirma otro de los entrevistados, "lo que hace es entregar su propio conocimiento a la disposición de los estudiantes, cuanto más generosa sea esa entrega y más ordenada yo creo que mejores son los resultados" (entrevista 6). Por lo tanto, tiene una labor importantísima en el desarrollo del aprendizaje, pues lo sistematiza, lo vuelve asequible, no obstante, este solo podrá darse si los estudiantes están motivados y cumplen con sus obligaciones.

A partir de las teorías implícitas estudiadas, se puede reconocer una importante influencia de la teoría interpretativa en las representaciones de los docentes sobre lo que significa enseñar; en ella se expone que el conocimiento puede lograrse a partir de la ejecución de unos procesos mentales y el favorecimiento de ciertas condiciones, en las cuales el docente tiene injerencia; debido a que su propia preparación influirá en el aprendizaje. Igualmente se destaca la importancia del esfuerzo del estudiante para poder asimilar los contenidos.

Los docentes tienen en común un gran interés en promocionar la reflexión y el análisis sobre su campo de estudio, de tal manera que los conocimientos no sean mecánicos sino que los estudiantes puedan dar opiniones bien fundamentadas. Esto parece indicar una preocupación por procesos mentales superiores, lo cual revelaría una inclinación más fuerte hacia la teoría interpretativa que a la directa.

La segunda categoría de análisis responde a las estrategias que utilizan los profesores como resultado de un proceso de planificación intencional de su práctica docente, tomando en consideración el contexto y características de los contenidos a enseñar (Pozo, Monereo y Castello, 2001).

Los docentes aluden a una planificación a partir del contenido y tipo de cursos que estén impartiendo; así, por ejemplo, toman en consideración si el contenido por abarcar se presta para realizar ejercicios, lecturas, exposiciones u otro tipo de actividades. El participante 5 dice lo 
siguiente: "creo que la metodología cambia totalmente de un curso a otro". Resulta interesante que la mayoría de actividades de clase coinciden en todos los docentes: exposición magistral para cursos teóricos, explicación y resolución de ejercicios para aquellos que son teórico prácticos.

Además de utilizar preferentemente la clase magistral, los participantes mencionaron que cambian la clase participativa por la primera debido a un fallo en la estrategia seleccionada previamente. Sin embargo, cabe destacar que dos de los entrevistados sí hicieron alusión a estrategias de enseñanza. Uno de ellos manifiesta:

Trato de implementar estrategias distintas debido a tal vez el grupo en el que me encuentre en un determinado momento. Todos los años hago una revisión, si doy un curso por segunda vez, lo reviso, cambio cosas, me pongo a hacer un balance de cómo han sido las cosas el año pasado y digo: "bueno, esto no funcionó, lo puedo cambiar por esto otro, esto la verdad es que tampoco funcionó vamos a poner esto en lugar de, o vamos a hacer una cronología distinta, es decir, una cosa más difícil es mejor verla al comienzo para dejar al final cuando ya la gente está muy cansada o agotada tal vez un tema más ameno, menos pesado, con menos contenido" y así sucesivamente... (Entrevista 5).

Esta revisión revela una planificación en función de ciertos objetivos, contenidos y contextos. Este mismo docente aseguró que durante la planificación:

Si son ejercicios, yo los resuelvo todos en casa y me hago preguntas que me podrían hacer mis estudiantes y me pregunto cómo contestaría, siempre, a mí no me gusta llegar y que me hagan preguntas y ver que yo no he preparado eso; si es teoría hago un esquema, un mapa conceptual de lo que voy a decir en la clase.

Los datos obtenidos muestran mucha preocupación por las particularidades de los contenidos y cómo hacerlos llegar a los estudiantes. Uno de los participantes, al ser consultado sobre sus prácticas pedagógicas reveló que: "lo que yo hago es enseñar con el ejemplo, es decir, con el ejemplo de la realidad, si vamos a estudiar la lengua por qué la tenemos que estudiar como si fuera un elemento químico que 
yo meto en una probeta y la analizo, es decir, debemos estudiarla en su esencia" (Entrevista 4).

La naturaleza de la disciplina influye en la selección de una determinada manera de presentar los contenidos, no obstante, hace falta considerar también el contexto y los objetivos de aprendizaje. Como ya se dijo, las estrategias planificadas a veces son abortadas por la reacción de los estudiantes, por lo tanto, podría tratarse de un incidente crítico que afecta directamente la identidad profesional:

Muchas veces me vuelvo bastante magistral y no solamente tiene que ver con que me gusta... creo que me sentía cómodo con esas clases y aprendí de algunas de esas clases mucho; sino también porque esa pasividad del estudiante me lleva a mí mismo; porque si quiero hacer una clase participativa con un tema que me llevaría... hay un tema a desarrollar de media hora, si la hago participativa se me van las tres horas y no es porque la gente participe mucho sino sacándoles con cuchara todo, si la hago magistral me lleva media hora. (Entrevista 2).

Se puede deducir, entonces, que a pesar de la existencia de un proceso de planificación por parte de los docentes se debe trabajar aún más en la consideración de todos los factores que influyen en el aprendizaje para desarrollar estrategias en el sentido que se ha dado a este concepto en la teoría ya que no solamente se debe considerar el contenido y naturaleza del curso sino también el contexto (incluyendo a los estudiantes).

La tercera categoría se refería a los factores afectivos y motivacionales que influyen en la práctica pedagógica. A partir de los instrumentos aplicados, se recogen los sentimientos experimentados por los docentes y los principales factores que intervienen.

Los sentimientos negativos predominantes fueron: frustración, molestia, desmotivación, desgano; mientras que los positivos: orgullo, satisfacción, bienestar, alivio. Los primeros se experimentan, según confesaron los entrevistados, a raíz del comportamiento de los estudiantes. En las entrevistas los docentes afirmaron que la práctica profesional:

Tiene sus bemoles, sus inseguridades; a veces uno cuando tiene algún problema concreto, siente que no vale la pena lo que uno está haciendo, o que la gente no lo aprecia, tal vez cuando uno 
está en la clase y hay algunas actitudes de algunos estudiantes uno se le baja; pero después cuando uno con los años se encuentra a estudiantes y hacen algún comentarios sobre la labor que uno hizo o no es necesario que le digan nada, con solo verlos ubicados en un puesto y desempeñándose como profesionales, yo creo que yo me siento satisfecho. (Entrevista 3).

Los sentimientos positivos se producen luego de concluir la clase si todo ha salido como estaba previsto, o bien, al comprobar que su esfuerzo como profesores ha traído como consecuencia la superación de los estudiantes.

Bien que mal, es muy rico que un estudiante que tuviste hace 7 años te lo encontrás en la calle y te diga "idiay profe!", solo eso: "idiay, profe!", ¿verdad?, como que te conozcan a partir de tu identidad profesional, es como bonito también. (Entrevista 2).

Los factores que intervienen negativamente en los docentes son: la actitud de los estudiantes, la burocracia universitaria, las malas relaciones con compañeros. Ejercen una influencia positiva la actitud de los estudiantes y los logros en el campo de la investigación. Frente a lo que se considera un comportamiento inadecuado de los dicentes los participantes reaccionan fuertemente:

Entonces dependiendo de qué tan grave considere yo eso, puede ser que sí les eche la hablada sobre la falta de responsabilidad y la falta de compromiso con su formación. O puede simplemente que entonces yo asuma también una actitud de bueno diay que a mí qué me importa... Esa falta de compromiso, esa irresponsabilidad me desmotiva muchísimo y ahí es donde a veces tal vez hasta me agarro de la investigación un poco como para aliviar esa carga emocional tan pesada. (Entrevista 2).

Por lo tanto, se puede apreciar que los factores afectivos tienen gran incidencia en el desarrollo de las prácticas pedagógicas, pues en algunos casos orientan la selección de estrategias para la enseñanza y tienen injerencia en el trato del docente hacia el estudiante. 
La valoración social de la profesión también media para que se produzcan emociones positivas o negativas en los docentes. Igualmente, ocurrirá lo mismo con el ambiente en el cual se labora, la motivación y el reconocimiento. Por lo tanto, para lograr cambios positivos en la dimensión afectiva no solo son necesarias acciones de los individuos, sino también de las instituciones y del gremio.

Marchesi (2007) afirma:

El bienestar emocional es una condición necesaria para la buena actividad educativa. Hay que sentirse bien para educar bien, aunque el bienestar emocional debe ir acompañado del saber y de la responsabilidad moral para que la actividad docente alcance su madurez. (p. 141).

Para impedir que las emociones negativas lleven al docente a una situación de quiebre emocional o bien interferir inadecuadamente en su práctica profesional, tiene que existir un trabajo en consenso por parte de los profesores y de las autoridades administrativas de su lugar de trabajo.

Se ha visto cómo los aspectos de la identidad profesional tienen relación directa con las prácticas pedagógicas, pues todos influyen en la manera cómo se desempeña el docente. En primer lugar, parten de la concepción sobre el aprendizaje y, por ende, marcan su papel en la relación que el estudiante tiene con este; en segundo lugar, esas concepciones y los sentimientos asociados a la práctica determinan la selección de estrategias de mediación, las cuales a su vez tendrán una repercusión en el ambiente de clase y podrían producir nuevos sentimientos que continúen alimentando el círculo.

\section{Conclusiones y recomendaciones}

Como conclusión general se puede afirmar que los aspectos de la identidad profesional docente efectivamente tienen implicaciones fuertes en las prácticas pedagógicas del profesorado del Departamento de Lingüística de la Escuela de Filología, Lingüística y Literatura. Por lo tanto, es posible tener un mejor conocimiento de dichas prácticas a partir del análisis sobre la identidad realizado.

Con respecto al primer objetivo específico, el cual buscaba determinar las concepciones que posee la población estudiada sobre cómo se 
enseña y aprende su materia, permitió determinar que existe una fuerte conexión entre las ideas expresadas por los docentes y la teoría interpretativa del aprendizaje, cuyo planteamiento principal es que hay procesos mentales entre la presentación de los contenidos y el aprendizaje, el cual se logrará a través del esfuerzo del estudiante, además de la supresión de los distractores.

Los docentes también poseen una serie de concepciones sobre los retos y demandas de su profesión. Para ellos es fundamental propiciar un espíritu crítico, nuevamente encontramos un punto de enlace con respecto a la teoría interpretativa, en este caso en sus versiones más elaboradas, en las cuales se comprende la labor del estudiante en términos de procesos mentales complejos.

Se concluye del segundo objetivo, el cual remitía a la mención de las estrategias utilizadas por los docentes, que desde el concepto de estrategia propuesto no se podría hablar de un uso adecuado, pues muchos de los profesores respondieron que tienen una marcada predilección por un tipo particular de clase (magistral), lo cual lleva a inferir que no adecuan en función de elementos como contexto, objetivos y contenidos; por lo tanto, no emplean procedimientos estratégicos.

El último objetivo de la investigación proponía describir las implicaciones de los factores afectivos en su práctica profesional. Los sentimientos que influyeron mayormente en los docentes fueron la frustración y el enojo a raíz del comportamiento de sus estudiantes o su actitud frente al curso. Los sentimientos positivos fueron el orgullo, la seguridad y la satisfacción que causa la labor docente. Es importante mencionar que las emociones afloran principalmente por la relación con los estudiantes, componente fundamental del trabajo académico. Pero también influye la valoración social de la profesión.

A partir de los datos analizados se pueden establecer una serie de recomendaciones. En primer lugar, se debe reconocer que este estudio es apenas un trabajo exploratorio, por lo tanto, sería aconsejable realizar trabajos posteriores para profundizar en esta línea de investigación. Por ejemplo, sería aconsejable un trabajo de investigación-acción participativa que permita implementar y evaluar una propuesta de capacitación en didáctica para la enseñanza de la Lingüística, la Gramática y la Expresión Escrita (áreas de Filología en las que interviene el Departamento de Lingüística). 
De igual manera se recomienda a los docentes participantes buscar asesoría en el ámbito de la didáctica para así fortalecer su práctica profesional. No solo mediante la inclusión de las TIC sino también capacitación sobre enseñanza estratégica y evaluación auténtica.

Una vez explicitados y analizados los aspectos de la identidad profesional docente se hace más fácil mejorar aquellos que están débiles. Por ejemplo, los factores afectivos, pues es posible investigar en el campo de la competencia emocional y a partir de allí buscar maneras de minimizar la incidencia negativa.

Por último, es recomendable que la Escuela de Filología, Lingüística y Literatura trabaje en aquellos factores que afectan negativamente la práctica docente $\mathrm{y}$, que dada su naturaleza, puedan manejarse institucionalmente; por ejemplo, las relaciones entre docentes o los canales de comunicación.

\section{Referencias bibliográficas}

Álvarez, M., Arely, A., Gazel, A., Gutiérrez, K. y Vargas, A. (2010). Percepciones del personal docente en educación inicial sobre su profesión en el papel que desempeñan en la sociedad. (Tesis de licenciatura no publicada). Universidad de Costa Rica: San José.

Cisterna, F. (2005). Categorización y triangulación como procesos de validación del conocimiento en investigación cualitativa. Theoría, 14 (001), 61-71. Recuperado de http://redalyc.uaemex.mx/pdf/299/29900107.pdf

Contreras, C. (setiembre, 2010). Investigando en la identidad profesional docente: Los profesores universitarios y los incidentes críticos que ocurren en sus aulas. Trabajo presentado en Congreso Iberoamericano de Educación: Metas 2021. Recuperado de http://www.chubut.edu.ar/descargas/secundaria/congreso/DOCENTES/R0770_Contreras.pdf

Contreras, C., Monereo, C. y Badia, A. (2010). Explorando en la identidad: ¿Cómo enfrentan los docentes universitarios los incidentes críticos que ocurren en las aulas de formación de futuros profesores? Revistas Electrónicas UACh 36 (2), 63-81. Recuperado de http://www.scielo.cl/scielo. php?pid=S0718-07052010000200004\&script=sci_arttext

Cubero, Z. (1996). Percepción de factores de satisfacción del personal docente del área de Ciencias Sociales, Universidad de Costa Rica. (Tesis de maestría no publicada). Universidad de Costa Rica, San José.

Francis, S. (2012). Conciencia y ciencia en la labor del docente universitario. En Gutiérrez-Soto, M. y Piedra, L., Docencia constructivista en la universidad, (pp. 73- 105). San José, Costa Rica: Estación Experimental Fabio Baudrit.

Macchiarola, V. y Vogliotti, A. (setiembre, 2003). Teorías implícitas, innovación educativa y formación profesional de docentes. Trabajo presentado en el Congreso Latinoamericano de Educación Superior. Recuperado de http://conedsup.unsl. 
edu.ar/Download_trabajos/Trabajos/Eje_6_Procesos_Formac_Grado_PostG_ Distancia/Vogliotti\%20y\%20Otros.PDF

Marchesi, A. (2007). Sobre el bienestar de los docentes: Competencias, emociones y valores. Madrid, España: Editorial Alianza.

Marchesi, A. y Díaz, T. (s. f.). Las emociones y los valores del profesorado [versión Adobe Reader]. Recuperado de http://www.oei.es/valores2/Lasemocionesprofesorado.pdf

Monereo, C. (2010). La formación del profesorado: Una pauta para el análisis e intervención a través de incidentes críticos. Revista Iberoamericana de Educación (52), 149-178. Recuperado de http://www.rieoei.org/rie52a08.pdf

Monereo, C., Castelló, M., Clariana, M., Palma, M., Pérez, M. (1999). Estrategias de enseñanza y aprendizaje: Formación del profesorado y aplicación en la escuela [versión Adobe Reader]. Recuperado de http://www.terras.edu.ar/jornadas/119/biblio/79Las-estrategias-de-aprendizaje.pdf

Piedra, L. y Gutiérrez-Soto, M. (2012). Conciencia y ciencia en la labor del docente universitario. En Gutiérrez-Soto, M. y Piedra, L., Docencia constructivista en la universidad, (pp. 3-20). San José, Costa Rica: Estación Experimental Fabio Baudrit.

Pozo, I., Scheuer, N., Pérez, M., Mateos, M., Martín, E. y De la Cruz, M. (2006). Nuevas formas de pensar la enseñanza y el aprendizaje: Las concepciones de profesores y alumnos. Barcelona, España: Editorial Grao.

Pozo, J.I., Monereo, C. y Castelló, M. (2001). El uso del conocimiento estratégico. En Coll, C., Palacios, J. y Marchesi, A. (comps), Desarrollo psicológico y educación. Psicología de la educación escolar (pp. 211-258). Madrid, España: Alianza Editorial.

Venegas, M. E. (1995). La función docente en la Universidad de Costa Rica. El caso de las y los docentes de las áreas de Ciencias Sociales. (Tesis de maestría no publicada). Universidad de Costa Rica, San José. 